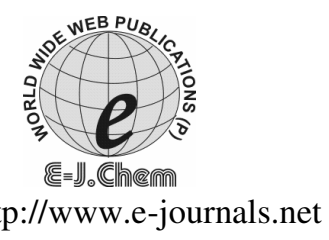

ISSN: 0973-4945; CODEN ECJHAO

E-Journal of Chemistry 2010, 7(2), 617-623

\title{
Synthesis and Studies of Novel Optically Active Schiff's Base Derivatives and their Antimicrobial Activities
}

\author{
ISHWAR J. PATEL ${ }^{*}$ and SHAILESH J. PARMAR \\ Department of Chemistry, \\ SIR. P. T. Sarvajanik College of Science, \\ Veer Narmad South Gujarat University, Surat 395017, Gujarat, India. \\ s_parmar1981@yahoo.com
}

Received 15 October 2009; Accepted 5 December 2009

\begin{abstract}
Several new Schiff's base derivatives were prepared by condensing various substituted benzaldehyde with 1-(4-aminophenyl)-2-\{4-[(S)-(4chlorophenyl)(phenyl) methyl]-1-piperazinyl $\}$ ethanone in presence of acid catalyst under reflux condition. All the compounds were characterized by elemental analysis and spectral studies. The newly synthesized compounds were evaluated for their antibacterial and antifungal activity.
\end{abstract}

Keywords: Schiff's base, Optically active, Chiral, Spectral studies, Specific optical rotation, Antibacterial activity and Antifungal activity.

\section{Introduction}

Schiff's base is a functional group or type of chemical compound containing a carbonnitrogen double bond with the nitrogen atom connected to an aryl group or an alkyl group but not hydrogen ${ }^{1}$. A Schiff's base (or azomethine) names after Hugo Schiff ${ }^{2}$. Schiff's bases can be synthesized from an aromatic amine and a carbonyl compound in a nucleophilic addition to a hemiaminal followed elimination of water to the imine ${ }^{3}$. Schiff's base ligands derived from salicylaldehyde and chiral amines have been widely applied in enantioselective cyclopropanation of styrenes ${ }^{4}$, asymmetric aziridination of olefins ${ }^{5}$, enantioselective epoxidation ${ }^{6}$, enantioselective ring opening of epoxides ${ }^{7}$, asymmetric oxidation of methyl phenyl sulfide ${ }^{8}$, enantioselective oxidation of silyl enol ${ }^{9}$ and trimethylsilylcyanation of benzaldehydes ${ }^{10}$. Schiff's base containing heterocycles have attracted much attention due to their diverse biological activity such as anticancer ${ }^{11}$, antiviral ${ }^{12-13}$, fungicidal ${ }^{14}$, bactericidal $^{15}$ and anti-HIV ${ }^{16}$. 


\section{Experimental}

All melting points were taken in open capillary tubes and are uncorrected. Specific optical rotations (SOR) were taken in Jasco digital polarimeter. Thin layer chromatography was performed on precoated TLC plates with silica gel (Merck $60 \mathrm{~F}_{254}$ ) and detection was done by UV lamp $(254 \mathrm{~nm})$. The IR spectra were obtained on a Simadzu FTIR-8400S spectrophotometer using $\mathrm{KBr}$ pellets. The ${ }^{1} \mathrm{H}$ NMR spectra in DMSO- $\mathrm{d}_{6}$ or $\mathrm{CDCl}_{3}$ were recorded on Bruker WM 400FT MHz spectrometer and chemical shift were reported as parts per million $(\delta \mathrm{ppm})$ down field using TMS as internal standard.
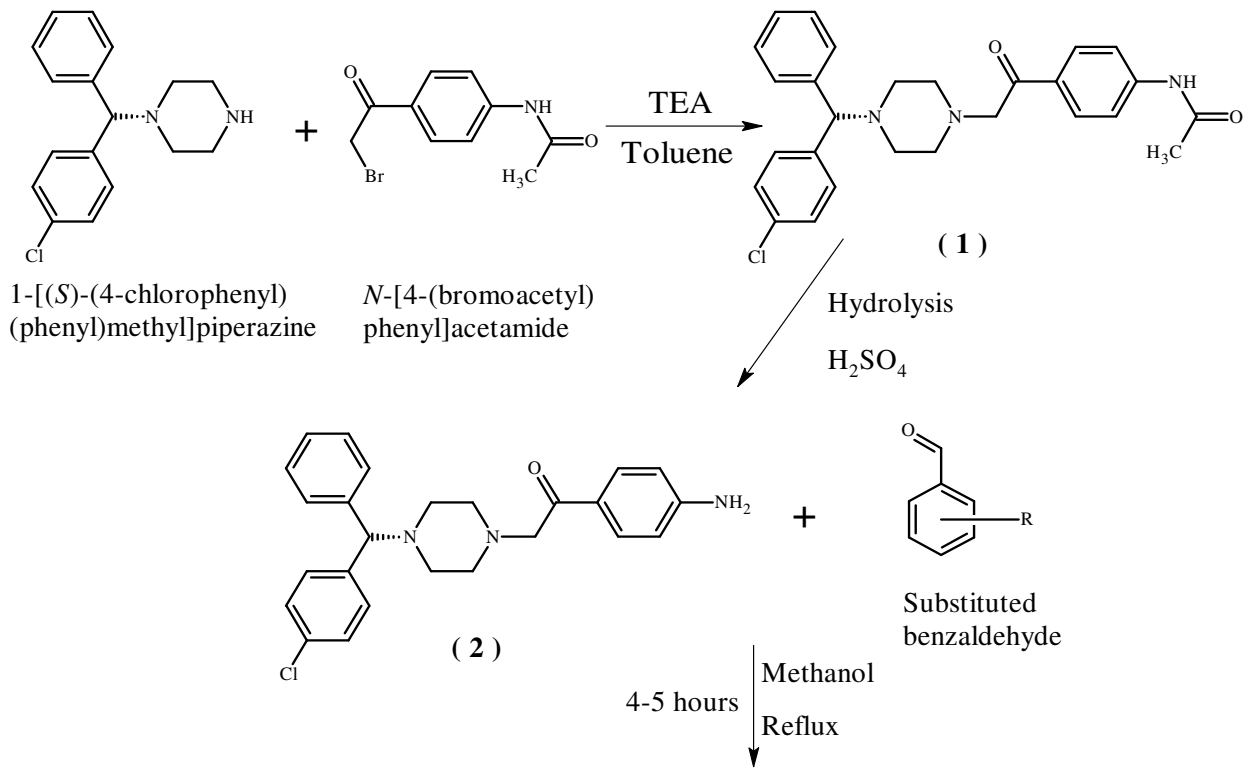

Substituted benzaldehyde

Where,
a) $\mathrm{R}=\mathrm{H}$
b) $\mathrm{R}=2-\mathrm{Cl}$
f) $\mathrm{R}=4-\mathrm{CH}_{3}$
c) $\mathrm{R}=4-\mathrm{Cl}$
g) $\mathrm{R}=3-\mathrm{NO}_{2}$
d) $\mathrm{R}=2-\mathrm{OCH}$
h) $\mathrm{R}=2-\mathrm{NO}_{2}$
e) $\mathrm{R}=4-\mathrm{OCH}$
i) $\mathrm{R}=2-\mathrm{OH}$
j) $\mathrm{R}=2-\mathrm{OH}-4-\mathrm{Br}$

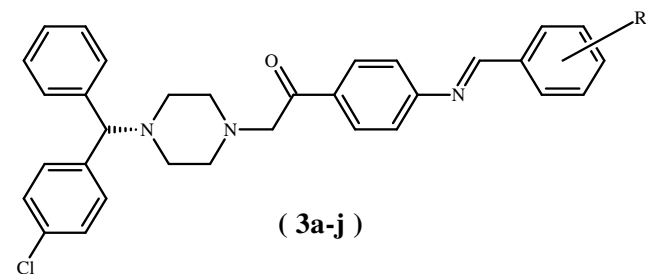

Preparation of $\mathrm{N}$-[4-(2-\{4-[(S)-(4-chlorophenyl)(phenyl)methyl]-1-piperazinyl\} acetyl) phenyl]acetamide(1)

A mixture of 1-[(S)-(4-chlorophenyl)(phenyl)methyl]piperazine (35 g, $0.122 \mathrm{~mol})$ [SOR:-20.18 ${ }^{\circ}$ and $N$-[4-(2-bromoacetyl)phenyl]acetamide (32 g, $\left.0.125 \mathrm{~mol}\right)$ was taken in methylene dichloride $(140 \mathrm{~mL})$. Triethylamine $(12.6 \mathrm{~g}, 0.125 \mathrm{~mol})$ was added drop wise below $20{ }^{\circ} \mathrm{C}$. The mixture was refluxed for $4-5 \mathrm{~h}$. After completion of reaction, it was cooled and washed with water. The organic mass was dry over sodium sulphate and solvent was removed. The product was recrystalized from methanol.

Yield: $86 \%$ MP: $75-76^{\circ} \mathrm{C}$ SOR: $-9.25^{\circ} \mathbf{I R}\left[v, \mathrm{~cm}^{-1}, \mathrm{KBr}\right]: 1666(\mathrm{C}=\mathrm{O}), 2956\left(-\mathrm{CH}_{2}\right)$, 757 (C-Cl). ${ }^{1} \mathbf{H}$ NMR [400 MHz, $\delta$, ppm, DMSO]: $2.12\left(3 \mathrm{H}, \mathrm{s},-\mathrm{COCH}_{3}\right), 3.36\left(2 \mathrm{H}, \mathrm{s},-\mathrm{COCH}_{2}\right)$, $10.61(1 \mathrm{H}, \mathrm{s},-\mathrm{NH}), 5.52(1 \mathrm{H}, \mathrm{s},-\mathrm{CH}-\mathrm{N}), 2.59-4.12\left(8 \mathrm{H}, \mathrm{m}, \mathrm{CH}_{2}\right.$ piperazine), 7.05-7.94 $(13 \mathrm{H}, \mathrm{m}, \mathrm{Ar}-\mathrm{H})$. 
Preparation of 1-(4-aminophenyl)-2-\{4-[(S)-(4-chlorophenyl)(phenyl)methyl]-1piperazinyl\}ethanone (2)

$N$-[4-(2-\{4-[(S)-(4-Chlorophenyl)(phenyl)methyl]-1-piperazinyl $\}$ acetyl)phenyl]acetamide (40 g, $0.087 \mathrm{~mol})$ was dissolve in methanol $(200 \mathrm{~mL})$. Sulfuric acid $(22 \mathrm{~g}, 0.22 \mathrm{~mol})$ was added drop wise by maintaining temperature below $15{ }^{\circ} \mathrm{C}$ with constant stirring. The reaction mixture was refluxed for $3 \mathrm{~h}$. After the completion of reaction, solvent was removed and added water $(300 \mathrm{~mL})$, made alkaline with concentrated ammonium hydroxide. The solid was separated and recrystalized from methanol.

Yield: $78 \% \mathrm{MP}: 110-112{ }^{\circ} \mathrm{C}$ SOR: $-5.45^{\circ} \mathrm{IR}\left[\mathrm{v}, \mathrm{cm}^{-1}, \mathrm{KBr}\right]: 1674(\mathrm{C}=\mathrm{O}), 3363$ $\left(-\mathrm{NH}_{2}\right), 758(\mathrm{C}-\mathrm{Cl}) .{ }^{1} \mathbf{H}$ NMR [400 MHz, $\left.\delta, \mathrm{ppm}, \mathrm{DMSO}\right]: 3.39\left(2 \mathrm{H}, \mathrm{s},-\mathrm{COCH}_{2}\right), 10.10$ $\left(2 \mathrm{H}, \mathrm{s},-\mathrm{NH}_{2}\right), 5.51(1 \mathrm{H},-\mathrm{CH}-\mathrm{N}), 2.56-4.09\left(8 \mathrm{H}, \mathrm{m}, \mathrm{CH}_{2}\right.$ piperazine $), 6.70-8.22$ $(13 \mathrm{H}, \mathrm{Ar}-\mathrm{H})$.

Preparation of 2-\{4-[(S)-(4-chlorophenyl)(phenyl)methyl]-1-piperazinyl\}-1-(4\{[aryl methylidene]amino\}phenyl)ethanone $(\mathbf{3 a}-\mathbf{j})$

A mixture of 1-(4-aminophenyl)-2-\{4-[(S)-(4-chlorophenyl)(phenyl)methyl]-1-piperazinyl $\}$ ethanone $(0.01 \mathrm{~mol})$ and substituted benzaldehyde $(0.01 \mathrm{~mol})$ in presence of glacial acetic acid $(0.5 \mathrm{~mL})$ in methanol $(25 \mathrm{~mL})$ was taken. It was refluxed gently for $4-5 \mathrm{~h}$. The solvent was removed and the product was isolated. It was recrystalized from acetic acid. The remaining compounds 3a-j was synthesized by using substituted benzaldehyde similarly. Their characterization data were recorded in Table 1.

Table 1. Characterization data of compounds (3a-j).

\begin{tabular}{|c|c|c|c|c|c|c|c|c|}
\hline \multirow{2}{*}{$\begin{array}{l}\text { Comp. } \\
\text { No. }\end{array}$} & \multirow[t]{2}{*}{$\mathrm{R}$} & \multirow{2}{*}{$\begin{array}{l}\text { Molecular } \\
\text { Formula, } \\
\text { (M.Wt) }\end{array}$} & \multirow{2}{*}{$\begin{array}{l}\mathrm{MP} \\
{ }^{\circ} \mathrm{C}\end{array}$} & \multirow{2}{*}{$\begin{array}{l}\text { SOR } \\
{[\alpha]_{d}^{\circ}}\end{array}$} & \multirow{2}{*}{$\begin{array}{c}\text { Yield } \\
\%\end{array}$} & \multicolumn{3}{|c|}{$\begin{array}{l}\text { Elementary Analysis } \\
\% \text { Found (Calculated) }\end{array}$} \\
\hline & & & & & & $\% \mathrm{C}$ & $\% \mathrm{H}$ & $\% \mathrm{~N}$ \\
\hline $1 \mathbf{a}$ & $\mathrm{H}$ & $\begin{array}{c}\mathrm{C}_{32} \mathrm{H}_{30} \mathrm{Cl} \mathrm{N} \mathrm{N}_{3} \mathrm{O} \\
(508)\end{array}$ & $138-139$ & -1.96 & 74 & $\begin{array}{c}75.61 \\
(75.65)\end{array}$ & $\begin{array}{c}5.92 \\
(5.95)\end{array}$ & $\begin{array}{c}8.25 \\
(8.27)\end{array}$ \\
\hline $1 b$ & $2-\mathrm{Cl}$ & $\begin{array}{c}\mathrm{C}_{32} \mathrm{H}_{29} \mathrm{Cl}_{2} \mathrm{~N}_{3} \mathrm{O} \\
(542)\end{array}$ & 204-205 & -2.15 & 79 & $\begin{array}{c}70.83 \\
(70.85)\end{array}$ & $\begin{array}{c}5.32 \\
(5.39)\end{array}$ & $\begin{array}{c}7.84 \\
(7.75)\end{array}$ \\
\hline $1 c$ & $4-\mathrm{Cl}$ & $\begin{array}{c}\mathrm{C}_{32} \mathrm{H}_{29} \mathrm{Cl}_{2} \mathrm{~N}_{3} \mathrm{O} \\
(542)\end{array}$ & $125-126$ & -1.90 & 73 & $\begin{array}{l}70.81 \\
(70.85)\end{array}$ & $\begin{array}{c}5.38 \\
(5.39)\end{array}$ & $\begin{array}{l}7.80 \\
(7.75)\end{array}$ \\
\hline $1 d$ & $2-\mathrm{OCH}_{3}$ & $\begin{array}{c}\mathrm{C}_{33} \mathrm{H}_{32} \mathrm{Cl} \mathrm{N}_{3} \mathrm{O}_{2} \\
\text { (538) }\end{array}$ & $179-180$ & -2.08 & 71 & $\begin{array}{c}73.69 \\
(73.66)\end{array}$ & $\begin{array}{c}5.95 \\
(5.99)\end{array}$ & $\begin{array}{c}7.85 \\
(7.81)\end{array}$ \\
\hline $1 \mathrm{e}$ & $4-\mathrm{OCH}_{3}$ & $\begin{array}{c}\mathrm{C}_{33} \mathrm{H}_{32} \mathrm{Cl} \mathrm{N}_{3} \mathrm{O}_{2} \\
\text { (538) }\end{array}$ & $148-150$ & -2.19 & 66 & $\begin{array}{l}73.65 \\
(73.66)\end{array}$ & $\begin{array}{c}5.98 \\
(5.99)\end{array}$ & $\begin{array}{c}7.80 \\
(7.81)\end{array}$ \\
\hline 1f & $4-\mathrm{CH}_{3}$ & $\begin{array}{c}\mathrm{C}_{33} \mathrm{H}_{32} \mathrm{Cl} \mathrm{N}_{3} \mathrm{O} \\
\text { (522) }\end{array}$ & $141-142$ & -3.10 & 75 & $\begin{array}{c}75.90 \\
(75.92)\end{array}$ & $\begin{array}{c}5.17 \\
(6.18)\end{array}$ & $\begin{array}{c}8.01 \\
(8.05)\end{array}$ \\
\hline $1 g$ & $3-\mathrm{NO}_{2}$ & $\underset{(553)}{\mathrm{C}_{32} \mathrm{H}_{29} \mathrm{Cl} \mathrm{N}_{4} \mathrm{O}_{3}}$ & $146-147$ & -3.58 & 72 & $\begin{array}{c}69.45 \\
(69.49)\end{array}$ & $\begin{array}{c}5.26 \\
(5.29)\end{array}$ & $\begin{array}{l}10.09 \\
(10.1)\end{array}$ \\
\hline $1 \mathrm{~h}$ & $2-\mathrm{NO}_{2}$ & $\begin{array}{c}\mathrm{C}_{32} \mathrm{H}_{29} \mathrm{Cl} \mathrm{N}_{4} \mathrm{O}_{3} \\
(553)\end{array}$ & $171-172$ & -3.27 & 76 & $\begin{array}{c}69.47 \\
(69.49)\end{array}$ & $\begin{array}{c}5.22 \\
(5.77)\end{array}$ & $\begin{array}{l}10.02 \\
(10.1)\end{array}$ \\
\hline $1 \mathbf{i}$ & $2-\mathrm{OH}$ & $\begin{array}{c}\mathrm{C}_{32} \mathrm{H}_{30} \mathrm{Cl} \mathrm{N}_{3} \mathrm{O}_{2} \\
\text { (524) }\end{array}$ & $133-134$ & -1.89 & 69 & $\begin{array}{c}73.31 \\
(73.34)\end{array}$ & $\begin{array}{c}5.78 \\
(5.29)\end{array}$ & $\begin{array}{c}8.00 \\
(8.02)\end{array}$ \\
\hline $\mathbf{1 j}$ & $\begin{array}{l}4-\mathrm{OH} \\
3-\mathrm{Br}\end{array}$ & $\begin{array}{c}\mathrm{C}_{32} \mathrm{H}_{29} \mathrm{Br} \mathrm{Cl} \mathrm{N} \\
\mathrm{O}_{2}(603) \\
\end{array}$ & $156-158$ & -2.45 & 74 & $\begin{array}{c}60.30 \\
(60.32) \\
\end{array}$ & $\begin{array}{r}4.60 \\
(4.62) \\
\end{array}$ & $\begin{array}{r}6.15 \\
(6.21) \\
\end{array}$ \\
\hline
\end{tabular}


2-\{4-[(S)-(4-Chlorophenyl)(phenyl)methyl]-1-piperazinyl\}-1-(4-[phenylmethylidene]amino\} phenyl)ethanone(3a)

IR $\left[v, \mathrm{~cm}^{-1}, \mathrm{KBr}\right]: 1672(\mathrm{C}=\mathrm{O}), 1593(-\mathrm{C}=\mathrm{N}), 757(\mathrm{C}-\mathrm{Cl}) .{ }^{1} \mathbf{H}$ NMR [400 MHz, $\delta, \mathrm{ppm}$, DMSO]: $3.31\left(2 \mathrm{H}, \mathrm{s},-\mathrm{COCH}_{2}\right), 8.71(1 \mathrm{H}, \mathrm{s},-\mathrm{CH}=\mathrm{N}), 5.48(1 \mathrm{H}, \mathrm{s},-\mathrm{CH}-\mathrm{N}), 2.54-4.11$ $\left(8 \mathrm{H}, \mathrm{m}, \mathrm{CH}_{2}\right.$ piperazine), 7.04-7.89 (18H, $\left.\mathrm{m}, \mathrm{Ar}-\mathrm{H}\right)$.

1-(4-\{[(2-Chlorophenyl)methylidene]amino\}phenyl)-2-\{4-[(S)-(4-chlorophenyl)(phenyl) methyl]-1-piperazinyllethanone(3b)

IR $\left[v, \mathrm{~cm}^{-1}, \mathrm{KBr}\right]: 1681(\mathrm{C}=\mathrm{O}), 1595(-\mathrm{C}=\mathrm{N}), 774(\mathrm{C}-\mathrm{Cl}) .{ }^{1} \mathbf{H}$ NMR [400 MHz, $\delta, \mathrm{ppm}$, DMSO]: $3.33\left(2 \mathrm{H}, \mathrm{s},-\mathrm{COCH}_{2}\right), 8.80(1 \mathrm{H}, \mathrm{s},-\mathrm{CH}=\mathrm{N}), 5.42(1 \mathrm{H}, \mathrm{s},-\mathrm{CH}-\mathrm{N}), 2.58-4.17$ $\left(8 \mathrm{H}, \mathrm{m}, \mathrm{CH}_{2}\right.$ piperazine $), 7.05-7.85(17 \mathrm{H}, \mathrm{m}, \mathrm{Ar}-\mathrm{H})$.

1-(4-\{[(4-Chlorophenyl)methylidene]amino\}phenyl)-2-\{4-[(S)-(4-chlorophenyl)(phenyl) methyl]-1-piperazinyl]ethanone(3c)

IR $\left[v, \mathrm{~cm}^{-1}, \mathrm{KBr}\right]: 1678(\mathrm{C}=\mathrm{O}), 1597(-\mathrm{C}=\mathrm{N}), 768(\mathrm{C}-\mathrm{Cl}) .{ }^{\mathbf{1}} \mathbf{H}$ NMR [400 MHz, $\delta, \mathrm{ppm}$, DMSO]: $3.38\left(2 \mathrm{H}, \mathrm{s},-\mathrm{COCH}_{2}\right), 8.78(1 \mathrm{H},-\mathrm{CH}=\mathrm{N}), 5.44(1 \mathrm{H}, \mathrm{s},-\mathrm{CH}-\mathrm{N}), 2.59-4.18(8 \mathrm{H}, \mathrm{m}$, $\mathrm{CH}_{2}$ piperazine), 7.00-7.82 (17H, m, Ar-H).

2-\{4-[(S)-(4-Chlorophenyl)(phenyl)methyl]-1-piperazinyl\}-1-(4-\{[(2-methoxyphenyl) methylidene]amino\}phenyl)ethanone $(\mathbf{3 d})$

IR $\left[v, \mathrm{~cm}^{-1}, \mathrm{KBr}\right]: 1667(\mathrm{C}=\mathrm{O}), 1594(-\mathrm{C}=\mathrm{N}), 2827\left(\mathrm{Ar}_{-}-\mathrm{OCH}_{3}\right), 756(\mathrm{C}-\mathrm{Cl}) .{ }^{\mathbf{1}} \mathbf{H}$ NMR [400 $\mathrm{MHz}, \delta, \mathrm{ppm}, \mathrm{DMSO}]: 3.70\left(3 \mathrm{H}, \mathrm{s},-\mathrm{OCH}_{3}\right), 3.35\left(2 \mathrm{H}, \mathrm{s},-\mathrm{COCH}_{2}\right), 8.68(1 \mathrm{H},-\mathrm{CH}=\mathrm{N})$, $5.51(1 \mathrm{H}, \mathrm{s},-\mathrm{CH}-\mathrm{N}), 2.57-4.16\left(8 \mathrm{H}, \mathrm{m}, \mathrm{CH}_{2}\right.$ piperazine $), 6.96-7.85(17 \mathrm{H}, \mathrm{m}, \mathrm{Ar}-\mathrm{H})$.

2-\{4-[(S)-(4-Chlorophenyl)(phenyl)methyl]-1-piperazinyl\}-1-(4-\{[(4-methoxyphenyl) methylidene]aminolphenyl)ethanone $(\mathbf{3 e})$

IR $\left[v, \mathrm{~cm}^{-1}, \mathrm{KBr}\right]: 1675(\mathrm{C}=\mathrm{O}), 1588(-\mathrm{C}=\mathrm{N}), 2822\left(\mathrm{Ar}_{-}-\mathrm{OCH}_{3}\right), 755(\mathrm{C}-\mathrm{Cl}) .{ }^{\mathbf{1}} \mathbf{H}$ NMR [400 $\mathrm{MHz}, \delta$, ppm, DMSO]: $3.74\left(3 \mathrm{H}, \mathrm{s},-\mathrm{OCH}_{3}\right), 3.32\left(2 \mathrm{H}, \mathrm{s},-\mathrm{COCH}_{2}\right), 8.79(1 \mathrm{H}, \mathrm{s},-\mathrm{CH}=\mathrm{N})$, $5.49(1 \mathrm{H}, \mathrm{s},-\mathrm{CH}-\mathrm{N}), 2.57-4.10\left(8 \mathrm{H}, \mathrm{m}, \mathrm{CH}_{2}\right.$ piperazine), 6.90-7.87 (17H, m, Ar-H).

2-\{4-[(S)-(4-Chlorophenyl)(phenyl)methyl]-1-piperazinyl\}-1-(4-\{[(4-methylphenyl) methylidene]amino\}phenyl)ethanone(3f)

IR $\left[v, \mathrm{~cm}^{-1}, \mathrm{KBr}\right]: 1672(\mathrm{C}=\mathrm{O}), 1596(-\mathrm{C}=\mathrm{N}), 1332\left(\mathrm{Ar}-\mathrm{CH}_{3}\right), 757(\mathrm{C}-\mathrm{Cl}) .{ }^{\mathbf{1}} \mathbf{H}$ NMR [400 $\mathrm{MHz}, \delta$, ppm, DMSO]: $2.25\left(3 \mathrm{H}, \mathrm{s},-\mathrm{CH}_{3}\right), 3.34\left(2 \mathrm{H}, \mathrm{s},-\mathrm{COCH}_{2}\right), 8.71(1 \mathrm{H},-\mathrm{CH}=\mathrm{N}), 5.56$ $(1 \mathrm{H}, \mathrm{s},-\mathrm{CH}-\mathrm{N}), 2.55-4.13\left(8 \mathrm{H}, \mathrm{m}, \mathrm{CH}_{2}\right.$ piperazine), 7.01-7.85 $(17 \mathrm{H}, \mathrm{m}, \mathrm{Ar}-\mathrm{H})$.

2-\{4-[(S)-(4-Chlorophenyl)(phenyl)methyl]-1-piperazinyl\}-1-(4-\{[(3-nitrophenyl) methylidene Jaminol phenyl)ethanone $(\mathbf{3 g})$

IR $\left[v, \mathrm{~cm}^{-1}, \mathrm{KBr}\right]: 1692(\mathrm{C}=\mathrm{O}), 1587(-\mathrm{C}=\mathrm{N}), 1344\left(\mathrm{Ar}-\mathrm{NO}_{2}\right), 756(\mathrm{C}-\mathrm{Cl}) .{ }^{\mathbf{1}} \mathbf{H}$ NMR [400 $\mathrm{MHz}, \delta$, ppm, DMSO]: $3.36\left(2 \mathrm{H}, \mathrm{s},-\mathrm{COCH}_{2}\right), 8.64(1 \mathrm{H}, \mathrm{s},-\mathrm{CH}=\mathrm{N}), 5.52(1 \mathrm{H}, \mathrm{s},-\mathrm{CH}-\mathrm{N})$, 2.54-4.20 (8H, m, $\mathrm{CH}_{2}$ piperazine), 7.04-7.98 (17H, m, Ar-H).

2-\{4-[(S)-(4-Chlorophenyl)(phenyl)methyl]-1-piperazinyl\}-1-(4-\{[(2-nitrophenyl) methylidene Jaminol phenyl)ethanone $(\mathbf{3 h})$

IR $\left[\mathrm{v}, \mathrm{cm}^{-1}, \mathrm{KBr}\right]: 1695(\mathrm{C}=\mathrm{O}), 1583(-\mathrm{CH}=\mathrm{N}), 1349\left(\mathrm{Ar}-\mathrm{NO}_{2}\right), 752(\mathrm{C}-\mathrm{Cl}) .{ }^{\mathbf{1}} \mathbf{H}$ NMR [400 $\mathrm{MHz}, \delta$, ppm, DMSO]: $3.37\left(2 \mathrm{H}, \mathrm{s},-\mathrm{COCH}_{2}\right), 8.68(1 \mathrm{H}, \mathrm{s},-\mathrm{CH}=\mathrm{N}), 5.53(1 \mathrm{H}, \mathrm{s},-\mathrm{CH}-\mathrm{N})$, 2.56-4.17 (8H, m, $\mathrm{CH}_{2}$ piperazine), 7.02-8.08 (17H, m, Ar-H). 
1-(4-\{[(2-Hydroxyphenyl)methylidene]amino\}phenyl)-2-\{4-[(S)-(4-chlorophenyl)(phenyl) methyl]-1-piperazinyl)ethanone $(\mathbf{3 i})$

IR $\left[v, \mathrm{~cm}^{-1}, \mathrm{KBr}\right]: 1681(\mathrm{C}=\mathrm{O}), 1595(-\mathrm{C}=\mathrm{N}), 3374(-\mathrm{OH}), 754(\mathrm{C}-\mathrm{Cl}) .{ }^{1} \mathbf{H}$ NMR [400 MHz, $\delta$, ppm, DMSO]: $3.35\left(2 \mathrm{H}, \mathrm{s},-\mathrm{COCH}_{2}\right), 4.50(1 \mathrm{H}, \mathrm{s},-\mathrm{OH}), 8.86(1 \mathrm{H}, \mathrm{s},-\mathrm{CH}=\mathrm{N}), 5.50(1 \mathrm{H}$, $\mathrm{s},-\mathrm{CH}-\mathrm{N}), 2.59-4.15$ (8H, m, $\mathrm{CH}_{2}$ piperazine), 6.92-7.84 (17H, m, Ar-H).

1-(4-\{[(3-Bromo-4-hydroxyphenyl)methylidene]amino\}phenyl)-2-\{4-[(S)-(4-chlorophenyl) (phenyl)methyl]-1-piperazinyllethanone $(\mathbf{3 j})$

IR $\left[v, \mathrm{~cm}^{-1}, \mathrm{KBr}\right]: 1678(\mathrm{C}=\mathrm{O}), 1591(-\mathrm{C}=\mathrm{N}), 3364(-\mathrm{OH}), 763(\mathrm{C}-\mathrm{Cl}), 687(\mathrm{C}-\mathrm{Br}) .{ }^{1} \mathbf{H}$ NMR [400 MHz, $\delta, \mathrm{ppm}, \mathrm{DMSO}]: 3.41\left(2 \mathrm{H}, \mathrm{s},-\mathrm{COCH}_{2}\right), 4.46(1 \mathrm{H}, \mathrm{s},-\mathrm{OH}), 8.69(1 \mathrm{H}, \mathrm{s},-$ $\mathrm{CH}=\mathrm{N}), 5.40(1 \mathrm{H}, \mathrm{s},-\mathrm{CH}-\mathrm{N}), 2.59-4.15\left(8 \mathrm{H}, \mathrm{m}, \mathrm{CH}_{2}\right.$ piperazine), 6.95-7.98 (16H, m, Ar-H).

\section{Results and Discussion}

The structures of substituted Schiff's base 3a-j were confirmed by elemental analysis, IR and ${ }^{1} \mathrm{H}$ NMR spectra. IR spectra showed absorption band at $1596 \mathrm{~cm}^{-1}$ indicated the stretching vibration of $-\mathrm{CH}=\mathrm{N}$ (Schiff's base) which confirming the condensation of reactants. C-H stretching vibration of $-\mathrm{CH}_{2}$ appeared at $2956 \mathrm{~cm}^{-1}$ and $-\mathrm{C}=\mathrm{O}$ stretching appeared at $1666 \mathrm{~cm}^{-1}$ indicated which confirming the condensation of reactants. The other peaks of IR spectra prove the structure of Schiff's base derivatives. ${ }^{1} \mathrm{H}$ NMR spectrum displayed signals for the presence of one imine proton $(-\mathrm{CH}=\mathrm{N})$ at $8.70 \mathrm{ppm}(1 \mathrm{H}, \mathrm{s})$, one ketone group $\left(-\mathrm{CO}-\mathrm{CH}_{2}\right)$ at $3.39 \mathrm{ppm}(2 \mathrm{H}, \mathrm{s})$, which also confirms the condensation of reactants.

\section{Biological screening}

\section{Antibacterial activities}

Antibacterial activities of all the compounds were studied against gram-positive bacteria [Staphylococcus aureus (MTCC96), Streptococcus pyogenes (MTCC442)] and gramnegative bacteria [Escherichia coli (MTCC443), Pseudomonas aeruginosa (MTCC424)] by the broth dilution method. Stock solutions of the series of compounds were prepared in DMSO. Each synthesized drug was diluted obtaining 2000 microgram $/ \mathrm{mL}$ concentration, as a stock solution. Serial dilutions were prepared in primary and secondary screening. In primary screening $500 \mu \mathrm{g} / \mathrm{mL}, 250 \mu \mathrm{g} / \mathrm{mL}$ and $125 \mu \mathrm{g} / \mathrm{mL}$ concentrations of the synthesized drugs were taken. The active synthesized drugs found in this primary screening were further tested in a second set of dilution against all microorganisms. The drugs found active in primary screening were similarly diluted to obtain $100 \mu \mathrm{g} / \mathrm{mL}, 50 \mu \mathrm{g} / \mathrm{mL}, 25 \mu \mathrm{g} / \mathrm{mL}$, $12.5 \mu \mathrm{g} / \mathrm{mL}, 6.250 \mu \mathrm{g} / \mathrm{mL}, 3.125 \mu \mathrm{g} / \mathrm{mL}$ and $1.5625 \mu \mathrm{g} / \mathrm{mL}$ concentrations. Under similar condition using Gentamycin, Ampicillin, Chloramphenicol, Ciprofloxacin, and Norfloxacin as a standard for comparison control experiment was carried out.

An examination of the data reveals that all compounds showed antibacterial activity. The compounds $\mathbf{3 e}, \mathbf{3 j}$, were highly active against all four organisms employed. The compounds 3a, 3c, 3d, 3e, 3f, 3g and 3i were highly active against Escherichia coli (MTCC443) and Staphylococcus aureus (MTCC96). Results were presented in Table 2.

\section{Antifungal activities}

The compounds 3a-j were also screened for their antifungal activity against Candida albicans (MTCC227), Aspergillus niger (MTCC282) and Aspergillus clavatus (MTCC1323) at $2000 \mu \mathrm{g} / \mathrm{mL}$ concentration using broth dilution method. As shown in Table 2, the antifungal 
activity data clearly showed that the compound 3a-j having a good activity against Candida albicans but less active against Aspergillus niger and Aspergillus clavatus. The antifungal activity was compared with the known standard drugs greseofulvin, nystatin and the results were presented in Table 2.

Table 2. Antimicrobial activity of compounds (3a-j).

\begin{tabular}{|c|c|c|c|c|c|c|c|}
\hline \multirow{3}{*}{ Comp. } & \multirow{2}{*}{\multicolumn{4}{|c|}{$\begin{array}{l}\text { Antibacterial activity } \\
\text { Minimum bactericidal } \\
\text { concentration } \mu \mathrm{g} / \mathrm{mL}\end{array}$}} & \multirow{2}{*}{\multicolumn{3}{|c|}{$\begin{array}{l}\text { Antifungal activity } \\
\text { Minimum fungicidal } \\
\text { concentration } \mu \mathrm{g} / \mathrm{mL}\end{array}$}} \\
\hline & & & & & & & \\
\hline & S. $\mathrm{a}^{\mathrm{a}}$ & S.p $p^{b}$ & E. $c^{c}$ & P. $a^{d}$ & C. $a^{\mathrm{g}}$ & A. $n^{e}$ & A. $c^{f}$ \\
\hline 3a & 50 & 250 & 250 & 250 & 250 & 500 & 1000 \\
\hline $3 \mathbf{b}$ & 250 & 500 & 500 & 500 & 100 & 1000 & 1000 \\
\hline $3 c$ & 62.5 & 500 & 250 & 250 & 500 & 1000 & 1000 \\
\hline 3d & 100 & 100 & 100 & 500 & 250 & 500 & 1000 \\
\hline $3 \mathbf{e}$ & 200 & 250 & 62.5 & 125 & 500 & 1000 & 1000 \\
\hline 3f & 250 & 500 & 100 & 125 & 250 & 250 & 1000 \\
\hline $3 g$ & 50 & 500 & 250 & 250 & 500 & 1000 & 1000 \\
\hline $3 \mathbf{h}$ & 500 & 50 & 500 & 250 & 100 & 1000 & 1000 \\
\hline $3 \mathbf{i}$ & 250 & 500 & 100 & 125 & 500 & 1000 & 1000 \\
\hline $3 \mathbf{j}$ & 100 & 125 & 500 & 250 & $>1000$ & 1000 & $>1000$ \\
\hline Gentamycin & 0.05 & 1 & 0.25 & 0.5 & - & - & - \\
\hline Ampicillin & 100 & 100 & 250 & 100 & - & - & - \\
\hline Chloramphenicol & 50 & 50 & 50 & 50 & - & - & - \\
\hline Ciprofloxacin & 25 & 25 & 50 & 50 & - & - & - \\
\hline Norfloxacin & 10 & 10 & 10 & 10 & - & - & - \\
\hline Nystatin & - & - & - & - & 100 & 100 & 100 \\
\hline Greseofulvin & - & - & - & - & 500 & 100 & 100 \\
\hline
\end{tabular}

S. $a^{a}$ - Staphylococcus aureus(MTCC96), S. $p^{b}$-Streptococcus pyogenes(MTCC442), E.c $c^{c}$-Escherichia coli(MTCC443), P.a - Pseudomonas aeruginosa(MTCC441), A. $n^{e}$-Aspergillus niger(MTCC282), A. $c^{f}$-Aspergillus clavatus(MTCC1323), C. $a^{g}$ - Candida albicans(MTCC227).

\section{Conclusion}

The antimicrobial activity of $\mathbf{3 a - j}$ carried out against some strain bacteria. The results show that the prepared compounds were toxic against the bacteria. The comparison of the antibacterial and antifungal activity of these compounds with standard drugs show that the presence of nitro, methoxy and halogen $(-\mathrm{Cl})$ group in the phenyl ring increases the activity.

\section{Acknowledgments}

Authors are thankful to the Principal, Sir P.T. Sarvajanik College of Science, Surat for providing research facilities. Authors are also thankful to Praveen Laboratory Pvt. Ltd, Jolwa, Surat for providing chemicals. Thanks are also due to Cadila Healthcare Limited, Baroda and Microcare Laboratory, Surat for providing spectral data, elemental analysis and antimicrobial activity.

\section{References}

1. IUPAC Compendium of chemical terminology, 1995, 67, 1364.

2. Schiff H, Justus Liebigs Ann Chem., 1864, 131, 118-119.

3. Gallant A J, Patrick B O and MacLachlan M J, J Org Chem., 2004, 69(25), 8739. 
4. $\quad$ Li Z N, Liu G and Zheng Z, Tetrahedron, 2000, 56, 7187.

5. Kenneth J O, Shiow J W and Cynthia J B, Tetrahedron Lett., 1992, 33, 1001.

6. Jacobsen E J, Zhang W and Guler M L, J Am Chem Soc., 1991, 113, 6703.

7. Kim G J and Shin J H, Cat Lett., 1999, 63, 83.

8. Sasaki C, Nakajima K and Kojima M, Bull Chem Soc Jpn., 1991, 64, 1318.

9. Waldemar A, Rainer T and Veit R S, J Am Chem Soc., 1998, 120, 708.

10. Aratani T, Yoneyoshi Y and Nagase T, Tetrahedron Lett., 1975, 1707.

11. Das W A, Torusdal M D, Ren S and Lien E J, Antiviral Res., 1999, 44, 201.

12. Bolos C A and Kyriakidis D A, Met-Based Drugs., 1998, 5(6), 323.

13. Chohan Z H and Farooq M A, Syn React Inorg Met-Org Chem., 2001, 31(10), 1853.

14. Farrow W M, Hannaa C and Schaeler F W, J Am Pharm Assoc., 1954, 43, 370.

15. Bhadur S, Goel A K and Verma R S, J Indian Chem Soc., 1976, 53, 590.

16. Pandeya S N, Sriram D N and Enle D, Arzeeim Forsch., 2000, 50(1), 55. 


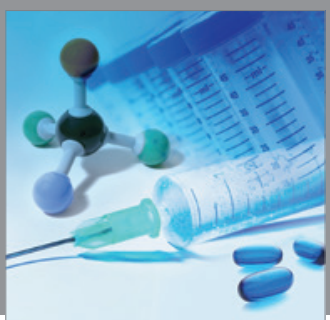

International Journal of

Medicinal Chemistry

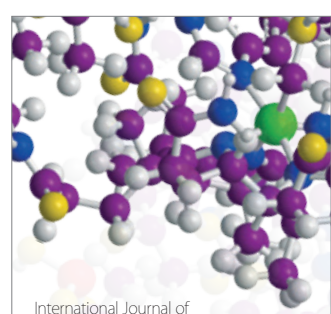

Carbohydrate Chemistry

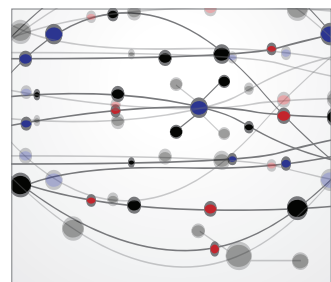

The Scientific World Journal
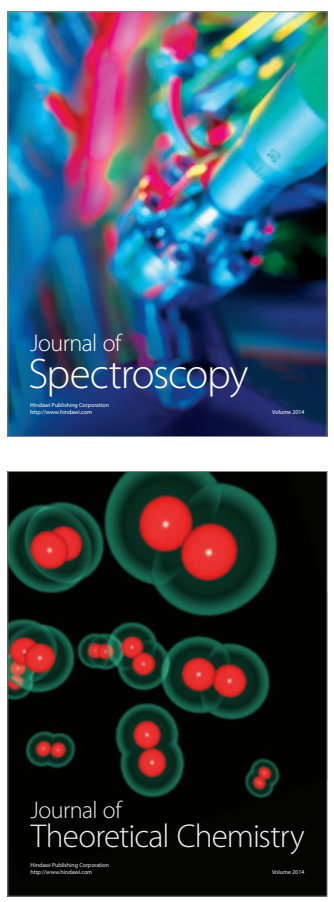
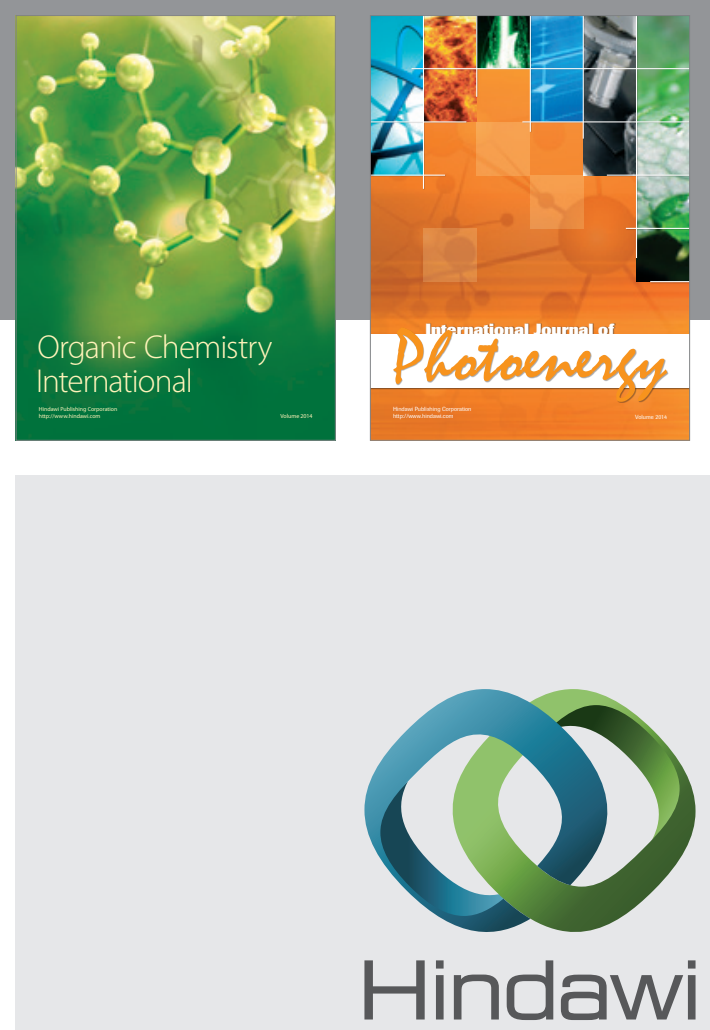

Submit your manuscripts at

http://www.hindawi.com
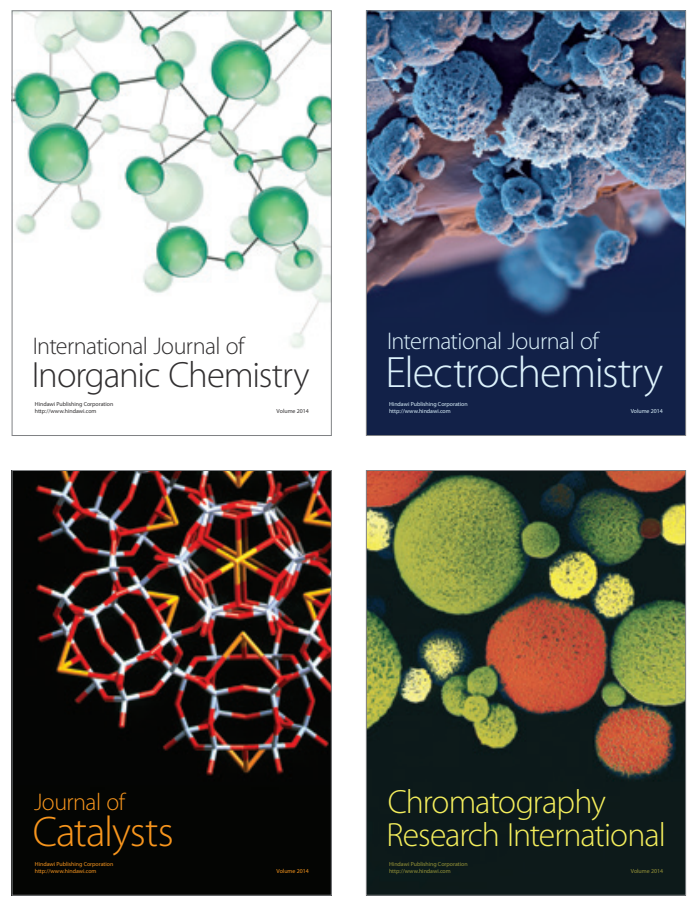
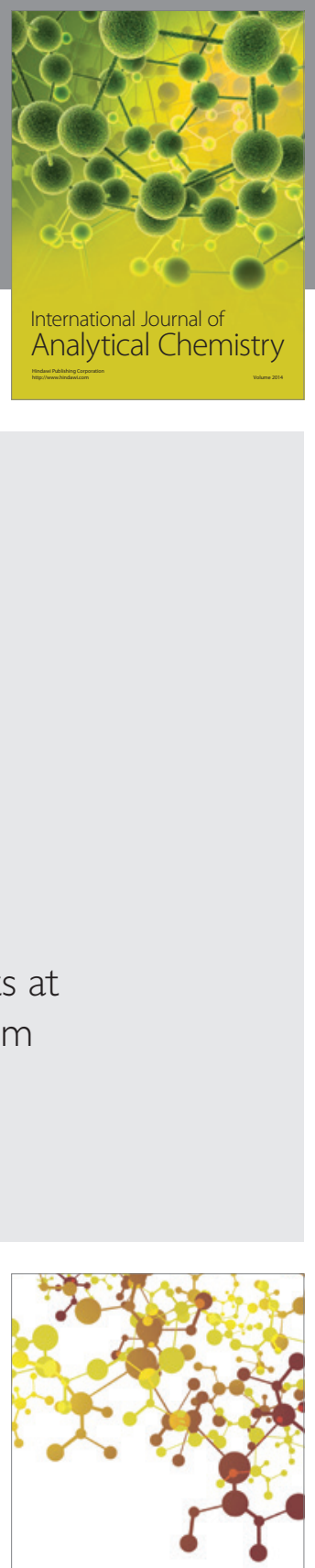

Journal of

Applied Chemistry
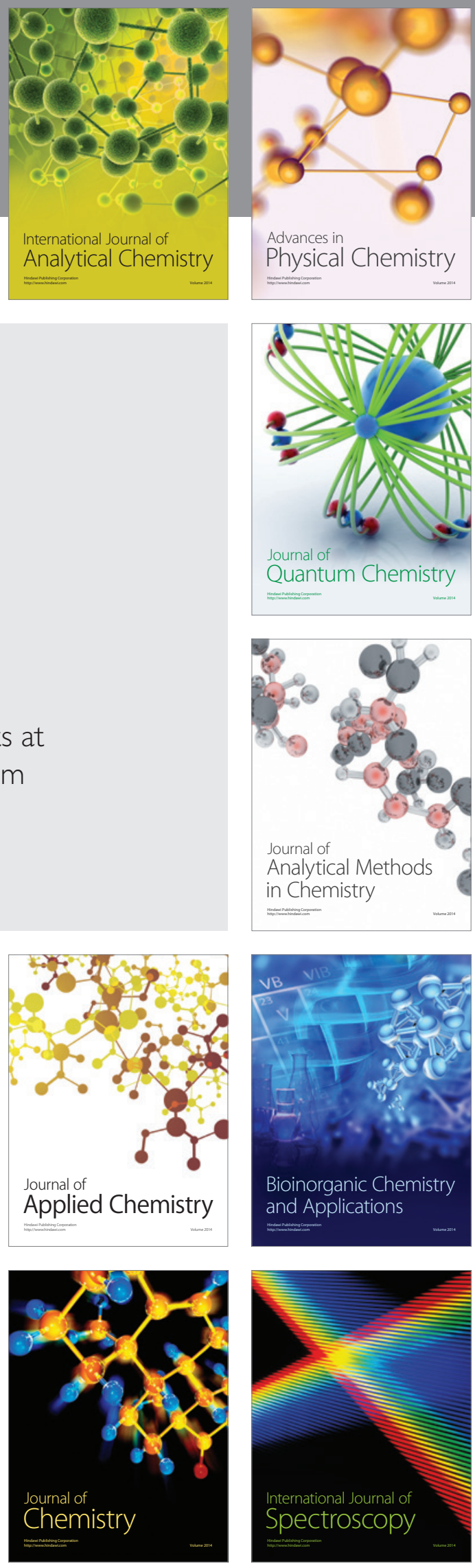